\title{
Maintenance of breast milk immunoglobulin A after high-pressure processing
}

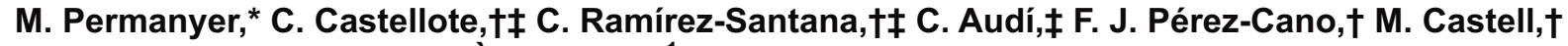 \\ M. C. López-Sabater, $¥ \S$ and À. Franch $¥ \ddagger^{1}$ \\ ${ }^{*}$ CENTA-IRTA, 17121 Monells, Girona, Spain \\ †Department of Physiology, Faculty of Pharmacy, University of Barcelona, Av. Joan XXIII s/n, 08028 Barcelona, Spain \\ ‡CIBER Epidemiología y Salud Pública (CIBERESP), 08003 Barcelona, Spain \\ $\S$ Department of Nutrition and Food Sciences, Faculty of Pharmacy, University of Barcelona, Av. Joan XXIII s/n, 08028 Barcelona, Spain
}

\section{ABSTRACT}

Human milk is considered the optimal nutritional source for infants. Banked human milk is processed using low-temperature, long-time pasteurization, which assures microbial safety but involves heat denaturation of some desirable milk components such as IgA. Highpressure processing technology, the subject of the current research, has shown minimal destruction of food macromolecules. The objective of this study was to investigate the influence of pressure treatments on IgA content. Moreover, bacterial load was evaluated after pressure treatments. The effects of high-pressure processing on milk IgA content were compared with those of low-temperature, long-time pasteurization. Mature human milk samples were heat treated at $62.5^{\circ} \mathrm{C}$ for 30 min or pressure processed at 400, 500, or $600 \mathrm{MPa}$ for 5 min at $12^{\circ} \mathrm{C}$. An indirect ELISA was used to measure IgA in human milk whey obtained after centrifugation at $800 \times g$ for $10 \mathrm{~min}$ at $4^{\circ} \mathrm{C}$. All 3 high-pressure treatments were as effective as low-temperature, long-time pasteurization in reducing the bacterial population of the human milk samples studied. After human milk pressure processing at $400 \mathrm{MPa}, 100 \%$ of IgA content was preserved in milk whey, whereas only $72 \%$ was retained in pasteurized milk whey. The higher pressure conditions of 500 and $600 \mathrm{MPa}$ produced $\mathrm{IgA}$ retention of 87.9 and $69.3 \%$, respectively. These results indicate that high-pressure processing at $400 \mathrm{MPa}$ for $5 \mathrm{~min}$ at $12^{\circ} \mathrm{C}$ maintains the immunological protective capacity associated with $\operatorname{IgA}$ antibodies. This preliminary study suggests that high-pressure processing may be a promising alternative to pasteurization in human milk banking.

Key words: immunoglobulin A, human milk banking, pasteurization, high-pressure processing

\footnotetext{
Received August 14, 2009.

Accepted November 27, 2009

${ }^{1}$ Corresponding author: angelsfranch@ub.edu
}

\section{INTRODUCTION}

The structural and cellular components of the immune system are present in neonates at term delivery. However, evidence suggests that the immune system is developmentally immature at this time and may not respond appropriately to environmental stimuli (Walker, 2002; Adkins et al., 2004). Among the various factors directly involved in the infant's mucosal defenses, IgA present in colostrum and milk offers passive protection for the gastrointestinal system. In neonates, maternal IgA is not absorbed, but it protects the mucosal surfaces from infection and has repercussions for the intestinal flora and immune system development (Zinkernagel, 2001; Hanson et al., 2003). Immunoglobulin A is the major effector component at the mucosal surfaces. At birth, neonates are hypogammaglobulinemic (Van de Perre, 2003) and are reliant on the protection provided by maternal antibodies during the first $12 \mathrm{mo}$ of life (Zinkernagel, 2001).

Breastfeeding is an unequaled way of providing the ideal food for healthy growth and development in infants; hence, worldwide public health recommendations advocate breastfeeding alone for the first 6 mo of life. Virtually all mothers can breastfeed, provided they have proper information and the support of their family and the health care system (WHO, 2003). When breastfeeding is not possible, pasteurized donor human milk is considered the best alternative to the mother's own milk, because it contains certain protective and developmental factors that are not found in infant formula (Wight, 2001; Hanson and Silfverdal, 2009).

In an increasing number of countries, milk-banking associations offer safe donor human milk to substitute mother's milk. Because microbial contamination can occur during collection and handling of human milk, the milk is pasteurized to prevent transmission of pathogens to the infant. The guidelines followed in human milk banks recommend processing donor milk by what is known as low-temperature, long-time (LTLT) pasteurization, a monitored process in which donor milk is heated at $62.5^{\circ} \mathrm{C}$ for $30 \mathrm{~min}$ (the temperature should 
not fall below this point) (HMBANA, 2005; REDBLHBR, 2005; Hartmann et al., 2007). The effects of LTLT and high-temperature, short-time pasteurization methods on $\operatorname{IgA}$ milk content have been studied. Human and animal Ig are thermolabile compounds, and heat denaturation has been observed after both pasteurization treatments (Braga and Palhares, 2007; Trujillo et al., 2007; Chantry et al., 2009).

Among the modern technologies currently used in the food industry, one of the most important involves nonthermal treatment and high-pressure processing (HPP). High-pressure processing is a promising method used to extend the shelf life of milk and other food products. In addition to inhibition or destruction of microorganisms, HPP has an influence on the physical and chemical properties of milk. Nevertheless, it seems that Ig denaturation occurs only at the highest pressures and at $50^{\circ} \mathrm{C}$ (Felipe et al., 1997). This observation led to the idea of applying HPP to breast milk as an alternative to heat treatment to preserve the Ig content (Trujillo et al., 2002). The aim of this study was to compare the effect of heat pasteurization and $3 \mathrm{HPP}$ treatments on breast milk IgA concentration to improve the immunological quality of this special product for infants. Moreover, bacterial load was evaluated after thermal and pressure treatments.

\section{MATERIALS AND METHODS}

\section{Milk Samples}

Mature human milk samples $(\mathrm{n}=10)$ were obtained from healthy volunteer donors of Asociación proLactancia de Barcelona (ALBA) in Barcelona (Spain). Informed consent to participate was obtained from all donors and the study was approved by the Ethics Review Board of the University of Barcelona. All women had delivered at term between 38 and $40 \mathrm{wk}$ of physiologic gestation. Data on the mother's weight increase during gestation, baby's birth weight, diet, any treatment received, and pregnancy history were systematically recorded. The main data recorded, expressed as the mean [SEM (range)], were maternal age, 33.9 yr [0.82 (29-38)], babies' mean BW, 3,150 g [183 $(2,650-4,120)]$, and time of lactation at which milk was collected, 113 d [24.6 (38-227)] after parturition. Only one woman delivered by caesarian section or had previous pregnancies. The exclusion criteria for mothers were smoking, immunodeficiency, malnutrition, recent local or systemic infectious disease, and psychiatric disorders.

Milk samples were collected in disposable sterilized flasks with an electric breast pump connected to a oneuse nipple adapter (Ameda Lactaline Doble, Austin,
TX) by emptying both breasts between 1000 and 1200 $\mathrm{h}$, to avoid changes due to circadian rhythms. Each milk sample was divided into several aliquots and randomly assigned to 1 of 6 groups. Two groups were assigned to LTLT pasteurization; 1 was immediately LTLT pasteurized (PM) and the other was not thermally treated (pasteurization control; raw human milk, RM). The remaining 4 groups were assigned to HPP treatment: 3 were pressurized at 400,500, and $600 \mathrm{MPa}$ (HPP-400, HPP-500, and HPP-600, respectively) and the other was not processed by HPP (reference group, HPPRef). All aliquots from the 6 groups, those related to LTLT pasteurization and those related to HPP, were stored at $-80^{\circ} \mathrm{C}$ until analysis or processing. A maximum of $6 \mathrm{~h}$ elapsed between collection and freezing.

\section{LTLT Pasteurization}

Samples from the PM group were heat-treated according to the LTLT pasteurization protocol (REDBLHBR, 2005). Briefly, sterilized glass tubes containing 10 $\mathrm{mL}$ of human milk were placed in a thermostatically controlled water bath under agitation at a constant temperature of $62.5^{\circ} \mathrm{C}$. Glass tubes containing bovine milk were used as blanks. Once the blanks reached $62.5^{\circ} \mathrm{C}$, samples were maintained in the water bath for 30 min under constant agitation. Following heat treatment, tubes were rapidly cooled in an ice bath. After pasteurization, samples were immediately aliquoted and stored at $-80^{\circ} \mathrm{C}$. Samples from the RM group were used as controls. Aliquots of both RM and PM samples were assigned for microbiological analysis.

\section{HPP}

For HPP treatment, appropriate amounts of milk samples were aseptically packed in specially made flexible polyethylene bags, vacuum-sealed to keep out air, and inserted into an additional individual bag made of the same material. Samples forming the HPP-400, HPP-500, and HPP-600 groups were pressurized for 5 min per batch at an isostatic pressure of 400,500 , and $600 \mathrm{MPa}$, respectively. Repeated pressure treatment was not used. One group of samples was thawed in the same way as the other HPP groups but was not processed by HPP; it served as the reference group (HPP-Ref). High-pressure processing was performed by using an industrial pressurization unit (Wave 6000/120 system, NC Hyperbaric, Burgos, Spain) with a maximum working pressure of $650 \mathrm{MPa}$ and a working volume of 120 $\mathrm{L}$, provided with a $300-\mathrm{mm}$ internal diameter horizontal layout vessel and 3 intensifiers. Cooled water without additives was used as the pressure transmitting fluid. The extent of adiabatic heating in the pressure unit 
was calculated according to the method of Balasubramaniam et al. (2004):

$$
\mathrm{T}_{\mathrm{f}}=\mathrm{T}_{\mathrm{i}}+(\alpha \cdot \mathrm{P}) / 100,
$$

where $T_{f}$ is the final temperature $\left({ }^{\circ} \mathrm{C}\right), \mathrm{T}_{\mathrm{i}}$ is the initial temperature $\left({ }^{\circ} \mathrm{C}\right), \alpha$ is the compression heating factor $\left({ }^{\circ} \mathrm{C} / \mathrm{MPa}\right)$, and $\mathrm{P}$ is the applied pressure. The initial water vessel temperature was set at $12^{\circ} \mathrm{C}$, and the adiabatic heating constant for water at $2.5^{\circ} \mathrm{C} / \mathrm{MPa}$ was used to calculate the extent of adiabatic heating in the pressure unit during processing. Final temperatures after $5 \mathrm{~min}$ at 400,500 , or $600 \mathrm{MPa}$ were $22.0,24.5$, and $27.0^{\circ} \mathrm{C}$, respectively. The pressure unit took 2 to 3 min to reach the desired pressure and less than $1 \mathrm{~min}$ to release the pressure.

After HPP treatment, all group samples (HPP-Ref, HPP-400, HPP-500, and HPP-600) were transferred to water for rapid cooling. One aliquot of all samples from each group was immediately analyzed for microbial content. The remaining aliquots were stored at $-80^{\circ} \mathrm{C}$ until obtainment of whey and quantitation of $\operatorname{IgA}$.

\section{Microbiological Analysis}

The microbiological quality of human milk samples was assessed by horizontal methods. Total microbial and Enterobacteriaceae counts were recorded. Aliquots of samples $(1 \mathrm{~mL})$ were mixed with $9 \mathrm{~mL}$ of $0.1 \%$ peptone water. For microbial enumeration, $1 \mathrm{~mL}$ of serial dilutions (1:10, diluent, $0.1 \%$ peptone water) of sample homogenate was used. Total viable counts were assessed on plate count agar medium (PCA Agar, Merck, Darmstadt, Germany) prepared according an internal protocol, with inclusion of $1 \mathrm{~mL}$ of homogenate in 15 $\mathrm{mL}$ of liquid culture medium, and left to stand at room temperature until solidification. Plates were incubated at $30 \pm 1^{\circ} \mathrm{C}$ for $72 \mathrm{~h}$. Enterobacteriaceae were counted using violet red bile glucose agar medium (VRBD Agar, Merck) prepared according an internal protocol, with inclusion of $1 \mathrm{~mL}$ of homogenate in $15 \mathrm{~mL}$ of liquid culture medium in a double layer. Plates were incubated at $30 \pm 1^{\circ} \mathrm{C}$ for $24 \mathrm{~h}$. The efficiency in reducing the total bacterial population of treatments was calculated as percentage of reduction related to raw milk counts $[100-(f \cdot 100) / i]$, where $f$ is bacterial population after treatment, and $i$ is bacterial population before treatment (Trujillo et al., 2007).

\section{Human Milk Whey}

Human milk whey for IgA quantification was obtained from all the sample groups. Briefly, the milk fatty layer and cellular elements were removed by centrifugation at $800 \times g$ for $10 \mathrm{~min}$ at $4^{\circ} \mathrm{C}$, and immediately after, the intermediate aqueous phase was carefully collected using sterile disposable syringes. The resulting translucent whey was aliquoted to avoid repeated freeze-thaw cycles and stored at $-80^{\circ} \mathrm{C}$ until analysis. The methods to obtain human milk whey are modifications of those described by other authors (Hawkes et al., 1999; 2002; Böttcher et al., 2003).

\section{IgA Quantitation}

Immunoglobulin A was measured in human milk whey using an ELISA quantitation kit from Bethyl Laboratories (Montgomery, TX), following the manufacturer's instructions. All milk whey samples were analyzed at a 1:4,000 dilution. Data were expressed as micrograms per milliliter of whey. Intraassay precision was assessed by the coefficient of variation $(\mathbf{C V})$ of 22 replicates of human milk whey carried out by the same operator under the same experimental conditions. Interassay precision was determined by the CV found when analyzing aliquots of a human milk whey sample on successive days over a 4 -wk period. Duplicate determinations were performed on each plate.

\section{Statistical Analysis}

Because antibody concentrations are not normally distributed (Filteau, 2009), a nonparametric test for paired samples was used. For the LTLT treatment results, only the Wilcoxon $T$-test was carried out. For the HPP statistical analysis, the Friedman test was applied, considering each pressure used as the independent variable. When pressure treatment had a significant effect on IgA concentration, the Wilcoxon T-test was performed. The Wilcoxon $T$-test was also used for LTLT and HPP treatments when values were expressed in percentages; the value obtained before treatment was considered $100 \%$. The SPSS 16.0 software package (SPSS Inc., Chicago, IL) was used for the statistical analyses. Differences were considered significant at $P$ values of $<0.05$. All data are expressed as the mean \pm SEM.

\section{RESULTS AND DISCUSSION}

\section{Validation Parameters}

The intra- and interassay CV of the IgA quantification method were 3.89 and $12.56 \%$, respectively. These values meet the acceptable standards of precision proposed by Horwitz (1982). There is increasing interest in measuring immune factors in human milk to 
A

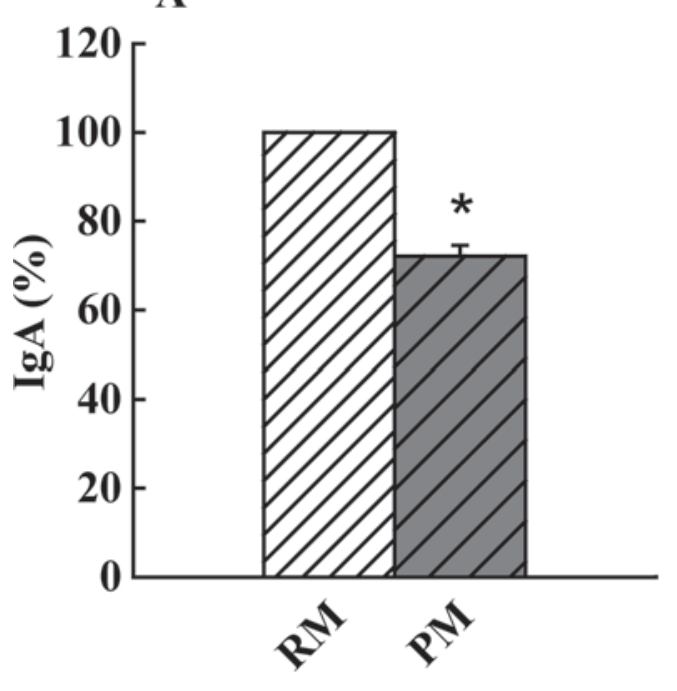

B

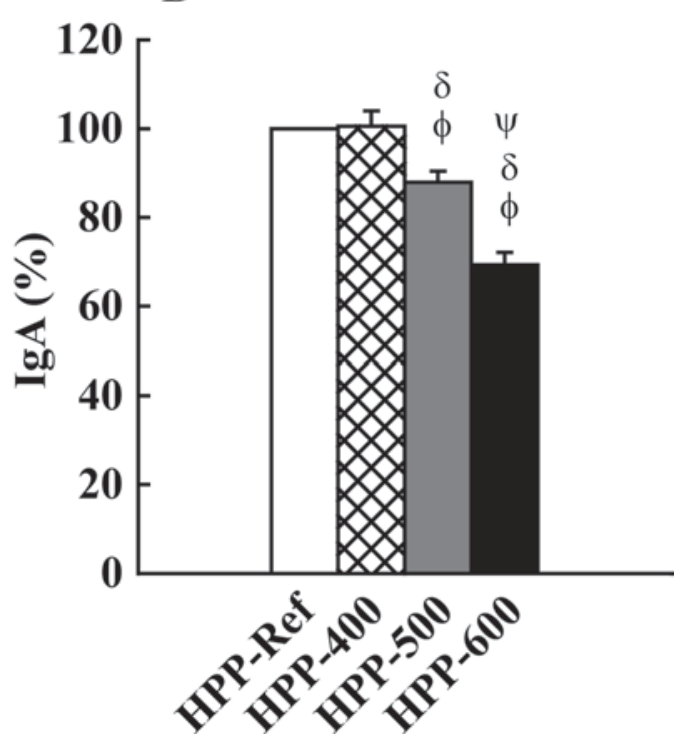

Figure 1. Percentage of IgA retention in human milk after (A) pasteurization and (B) HPP treatment. HPP = high-pressure processing; HPP-Ref $=0 \mathrm{MPa}$; HPP-400 $=400 \mathrm{MPa} ; \mathrm{HPP}-500=500 \mathrm{MPa} ; \mathrm{HPP}-600=600 \mathrm{MPa} ; \mathrm{PM}=$ pasteurized milk; RM $=$ raw, unprocessed milk. $* P$ $<0.01$ versus RM; $\phi$ versus HPP-Ref; $\delta$ versus HPP-400; $\psi$ versus HPP-500. Values represent the mean of 10 samples; vertical lines indicate the SEM.

investigate the effect of breastfeeding on maternal and infant health. Results generated from different laboratories are often difficult to compare because of method variations or insufficient description of the methods. The most commonly used assay to measure immune factors in human milk is ELISA. Nonetheless, breast milk is difficult to analyze by ELISA, likely because of its complex matrix. As has been recommended, it is important to describe in sufficient detail the sample preparation methods used and the quality control of the assay (Filteau, 2009). The IgA content in human milk in this study was measured only in the aqueous phase under the conditions described.

\section{Effect of LTLT Pasteurization on IgA Retention in Human Milk}

Banked human milk is pasteurized before distribution using LTLT thermal treatment. Pasteurization is necessary to inactivate pathogenic microorganisms but it also destroys or reduces many of the unique biological properties for which human milk is valued, such as some vitamins (Romeu-Nadal et al., 2008) and lysozyme (Viazis et al., 2007). Immunoglobulin A constitutes the main defense protein in human milk in terms of concentration and the breadth of its protective effects (Weaver et al., 1998). Whey milk IgA concentrations measured before pasteurization in this study ranged from 247 to $488 \mu \mathrm{g} / \mathrm{mL}$. Low-temperature, long-time pasteurization caused IgA loss in all samples and resulted in a difference in IgA concentration between RM and PM (338.45 \pm 27.03 vs. $243.64 \pm 19.98 \mu \mathrm{g} / \mathrm{mL} ; P<0.01)$.

Previous studies summarized by Ogundele (2000) showed that $\operatorname{IgA}$ is stable at $56^{\circ} \mathrm{C}$ but heat-labile at $62.5^{\circ} \mathrm{C}$ and destroyed by sterilization. Similarly, our data show that IgA content in human milk decreased after LTLT pasteurization, leaving IgA retention at $72.05 \%$ (Figure 1A). Thus, despite the considerable range of IgA content in human milk samples, the percentage of protein retention after LTLT treatment was similar in all samples. Furthermore, the percentage of IgA remaining after pasteurization was similar to that found in other studies using the same pasteurization method (Viazis et al., 2007; Czank et al., 2009). Of note, the recent study by Czank et al. (2009) confers critical importance to the temperature but not to the holding time for $\operatorname{Ig} \mathrm{A}$ retention.

\section{Effect of HPP Treatment on IgA Retention in Human Milk}

Over the last decade, there has been increasing interest in HPP treatment of foods. This nonthermal technology modifies the structure and functional properties of food macromolecules, such as proteins, without affecting the nutritional value. High-pressure processing is useful to prolong shelf life without producing detrimental changes in the processed products, as could be 
the case in milk banking. In addition, HPP is reported to be effective for microbial inactivation in animal milk (Trujillo et al., 2007).

In the present study, the HPP-Ref samples (i.e., raw milk samples that underwent the same freezethaw cycle as pressure-processed samples) showed an $\operatorname{IgA}$ concentration of $367.6 \pm 38.18 \mu \mathrm{g} / \mathrm{mL}$, a value similar to that seen in the RM samples. Therefore, in the present study, freeze-thawing did not induce IgA losses. Concentrations of IgA after pressure processing for $5 \mathrm{~min}$ at $12^{\circ} \mathrm{C}$ were $363.6 \pm 30.90 \mu \mathrm{g} / \mathrm{mL}$ for HPP$400,318.2 \pm 27.75 \mu \mathrm{g} / \mathrm{mL}$ for HPP-500, and $250.4 \pm$ $21.58 \mu \mathrm{g} / \mathrm{mL}$ for HPP-600. Although no changes in milk whey IgA content relative to HPP-Ref were found in the HPP-400 group, decreases were produced at the 2 higher pressures, HPP-500 $(P<0.01)$ and HPP-600 $(P<0.01)$, and there was a difference between HPP500 and HPP-600 $(P<0.01)$. These data, expressed in percentages, are shown in Figure 1B, with 100\% considered the mean value before pressure processing. As can be seen, processing at $400 \mathrm{MPa}$ produced no changes in IgA human whey milk concentration, whereas at 500 and $600 \mathrm{MPa}$, IgA retention was 87.93 and $69.31 \%$, respectively $(P<0.01)$.

Several studies have focused on HPP processing and Ig in bovine and goat milk, but to our knowledge, there is only one study on HPP treatment in human milk (Viazis et al., 2007). Those authors applied HPP at $400 \mathrm{MPa}$ for $0,30,60,90$, and $120 \mathrm{~min}$, and found that the shortest time interval resulted in $85 \% \operatorname{IgA}$ retention. In a study performed with goat milk, $10 \mathrm{~min}$ of treatment at 400 and $500 \mathrm{MPa}$ resulted in reductions in IgG content of 20 and 38\%, respectively (Trujillo et al., 2007). The Ig loss described in milk from humans and other mammals may be due to the lengthy time of pressure processing employed, which can also involve a higher temperature. In the study by Viazis et al. (2007), although sample temperature in the pressure vessel was controlled at a maximum of $31^{\circ} \mathrm{C}$, a progressive $\operatorname{IgA}$ decrease was observed over the processing period at $400 \mathrm{MPa}$, resulting in $75.4 \%$ of $\operatorname{IgA}$ retention at 120 min. However, pressure-induced denaturation at low to moderate pressures (100 to $400 \mathrm{MPa}$ ) may be reversible. Thus, as reported by Altuner et al. (2006), HPP at room temperature or mild heat disrupts relatively weak chemical bonds in milk, particularly hydrophobic bonds. This may be why some studies, in which HPP was applied for a longer time and at lower pressures than those used in the present study, showed no Ig loss in milk (Howlett et al., 1992; Felipe et al., 1997). Future studies are needed to clarify whether pressureassociated $\operatorname{Ig} \mathrm{A}$ loss is more homogeneous than antigenspecific, which could be beneficial for infant defenses.
This is related to the immunological quality of treated human milk.

\section{Effect of LTLT Pasteurization and HPP Treatment on Microbiological Quality}

The purpose of LTLT pasteurization of banked donor human milk is to eliminate potential pathogenic microorganisms and ensure microbial safety. In the present study, RM samples had a total microorganism count ranging from $1.3 \times 10^{2}$ to $2.9 \times 10^{4} \mathrm{cfu} / \mathrm{mL}$. These values are usually found in banked breast milk samples, and are within the range those accepted by the British Paediatric Association for donor milk banking (UKAMB, 2009). In 90\% of RM samples (9/10), Enterobacteriaceae were undetectable $(<10 \mathrm{cfu} / \mathrm{mL})$, and the only positive sample had $3.0 \times 10^{1} \mathrm{cfu} / \mathrm{mL}$. After LTLT pasteurization, the total bacterial population was undetectable, except for one sample that showed a level of $2 \times 10^{1} \mathrm{cfu} / \mathrm{mL}$. In the only positive $\mathrm{RM}$ sample, Enterobacteriaceae were also reduced to undetectable levels by heat treatment. Of note, the breast milk samples collected from volunteer donor mothers in the present study were likely less contaminated than those obtained from routine milk bank donors. Thus, it is not possible to assure effectiveness of the methods on routine banked milk with higher bacterial content. Serafini et al. (2003) reported that potentially pathogenic microorganisms were identified in $70.4 \%$ of the raw milk samples in a Brazilian milk bank, and even after pasteurization, $50.7 \%$ of the samples remained contaminated. Thus, future studies should determine whether HPP treatment is able to eliminate microorganisms in contaminated milk from routine donors.

The degree of microbial inactivation in human milk achieved by HPP was evaluated in the present study. The HPP treatment reduced the total bacterial and Enterobacteriaceae counts to undetectable levels regardless of the initial microbial level and the pressure used during processing. The 3 HPP treatments assayed at $12^{\circ} \mathrm{C}$ for 5 min were effective in reducing the bacterial population in the human milk samples to undetectable levels.

Despite the extensive research on the efficacy of HPP for inactivating microorganisms, the precise mechanism of pressure inactivation in vegetative cells is not completely understood. Moreover, depending on the suspending matrix and the effects of divalent cations such as $\mathrm{Ca}^{2+}$, the pressure resistance of microorganisms can vary (Hauben et al., 1998; Gervilla et al., 2000). Only one study in pathogen-inoculated milk has compared LTLT pasteurization and HPP treatment (Viazis et al., 2008). Thermal treatment and HPP processing at 400 
$\mathrm{MPa}$ for 0 to $50 \mathrm{~min}$ at $21^{\circ} \mathrm{C}$ to $31^{\circ} \mathrm{C}$ resulted in inactivation of bacterial pathogens, and the inactivation kinetics of some strains was more rapid in inoculated milk than in inoculated peptone. This may be because human milk contains some antimicrobial components (Ogundele, 2000).

\section{CONCLUSIONS}

To our knowledge, this is the first study that shows that high-pressure processing of human milk at 400 $\mathrm{MPa}$ for $5 \mathrm{~min}$ at $12^{\circ} \mathrm{C}$ resulted in $100 \%$ retention of milk whey IgA, whereas after LTLT pasteurization, only $72 \%$ of $\operatorname{IgA}$ was retained. To maintain IgA at 400 $\mathrm{MPa}$, a short processing time of $5 \mathrm{~min}$ and low temperature seem to be essential. In terms of $\operatorname{IgA}$ retention, HPP performed under these conditions provided better results than pasteurization, resulting in breast milk with the capacity to confer immunological protection on infants similar to that of unprocessed milk. As to the efficacy of HPP for inactivating microorganisms, excellent results were obtained herein, although further study with more highly contaminated human milk from routine milk bank donors should be performed to verify these findings. Additional studies would also be needed to explore the potential of HPP to retain other important immunologic and bioactive agents in human milk.

\section{ACKNOWLEDGMENTS}

This study was funded by grants from the Spanish Ministerio de Educación y Ciencia (AGL-2005-069401/ ALI) and Ministerio de Sanidad y Consumo (CIBER 06/02/0079) and from the Generalitat de Catalunya (SGCR-2005-00833). The authors are grateful to Olga Roig-Herrera (CENTA-IRTA, Monells, Girona, Spain) for her contribution in microbiology work, M. A. Canela (Departament de Matemàtica Aplicada i Anàlisi, Facultat de Matemàtiques, University of Barcelona, Spain) for his help in statistics, and Celine Cavallo for revising the English version of the manuscript. This research was possible thanks to the generosity of the human milk donors.

\section{REFERENCES}

Adkins, B., C. Leclerc, and S. Marshall-Clarke. 2004. Neonatal adaptative immunity comes of age. Nat. Rev. Immunol. 4:553564.

Altuner, E. M., H. Alpas, Y. K. Erdem, and F. Bozoglu. 2006. Effect of high hydrostatic pressure on physicochemical and biochemical properties of milk. Eur. Food Res. Technol. 222:392-396.

Balasubramaniam, V. M., E. Y. Ting, C. M. Stewart, and J. A Robbins. 2004. Recommended laboratory practices for conducting high-pressure microbial inactivation experiments. Innov. Food Sci. Emerg. Technol. 5:299-306.
Böttcher, M. F., M. C. Jenmalm, and B. Björkstén. 2003. Cytokine, chemokine and secretory $\operatorname{IgA}$ levels in human milk in relation to atopic disease and $\operatorname{IgA}$ production in infants. Pediatr. Allergy Immunol. 14:35-41.

Braga, L. P. M., and D. B. Palhares. 2007. Effect of evaporation and pasteurization in the biochemical and immunological composition of human milk. J. Pediatr. 83:59-63.

Chantry, C. J., K. Israel-Ballard, Z. Moldoveanu, J. Peerson, A. Coutsoudis, L. Sibeko, and B. Abrams. 2009. Effect of flash-heat treatment on immunoglobulins in breast milk. J. Acquir. Immune Defic. Syndr. 51:264-267.

Czank, C., D. K. Prime, B. Hartmann, K. Simmer, and P. E. Hartmann. 2009. Retention of the immunological proteins of pasteurized human milk in relation to pasteurizer design and practice. Pediatr. Res. 66:374-379.

Felipe, X., M. Capellas, and A. R. Law. 1997. Comparison of the effects of high-pressure treatments and heat pasteurization on the whey proteins in goat's milk. J. Agric. Food Chem. 45:627-631.

Filteau, S. 2009. Measuring trace immune factors in human milk. Pages 331-337 in Breast-Feeding: Early Influence on Later Health. G. Goldberg, A. Prentice, A. Prentice, S. Filteau, and K. Simondon ed. Advances in Experimental Medicine and Biology series, vol. 639. Springer, London, UK.

Gervilla, R., V. Ferragut, and B. Guamis. 2000. High-pressure inactivation of microorganisms inoculated into ovine milk of different fat contents. J. Dairy Sci. 83:674-682.

Hanson, L. A., M. Korotkova, and E. Telemo. 2003. Breast-feeding, infant-formulas, and the immune system. Ann. Allergy Asthma Immunol. 90(Suppl. 3):59-63.

Hanson, L. A., and S. A. Silfverdal. 2009. The mother's immune system is a balanced threat to the foetus, turning to protection of the neonate. Acta Paediatr. 98:221-228.

Hartmann, B. T., W. W. Pang, A. D. Keil, P. E. Hartmann, and K. Simmer. 2007. Best practice guidelines for the operation of a donor human milk bank in an Australian NICU. Early Hum. Dev. 83:667-673.

Hauben, K. J. A., K. Bernaerts, and C. W. Michiels. 1998. Protective effects of calcium on inactivation on Escherichia coli by using hydrostatic pressure. J. Appl. Microbiol. 85:678-684.

Hawkes, J. S., D.-L. Bryan, and R. A. Gibson. 2002. Variations in transforming growth factor beta in human milk are not related to levels in plasma. Cytokine 17:182-186.

Hawkes, J. S., D.-L. Bryan, M. J. James, and R. A. Gibson. 1999 Cytokines (IL-1beta, IL-6, TNF-alpha, TGF-beta1, and TGFbeta2) and prostaglandin $\mathrm{E}_{2}$ in human milk during the first three months postpartum. Pediatr. Res. 46:194-199.

HMBANA. 2005. Human Milk Banking Association of North America. http://hmbana.org.

Horwitz, W. 1982. Evaluation of analytical methods used for regulation of foods and drugs. Anal. Chem. 54:67A-76A.

Howlett, J. R., A. A. Ismail, D. W. Armstrong, and P. T. T. Wong. 1992. Pressure-induced conformational changes in an antigen and an antibody and the implications on their use for hyperbaric immunoadsorption. Biochim. Biophys. Acta 1159:227-236.

Ogundele, M. O. 2000. Techniques for the storage of human breast milk: implications for anti-microbial functions and safety of stored milk. Eur. J. Pediatr. 159:793-797.

REDBLH-BR. 2005. Red Nacional de Bancos de Leche Humana. BLHIFF/NT-34.05. http://fiocruz.br/redeblh/media/pasteurizaesp. pdf Accessed Jul. 21, 2009.

Romeu-Nadal, M., A. I. Castellote, A. Gayà, and M. C. López-Sabater. 2008. Effect of pasteurization on ascorbic acid, dehydroascorbic acid, tocopherols and fatty acids in pooled mature human milk. Food Chem. 107:434-438.

Serafini, A. B., M. C. D. P. B. André, M. A. V. Rodrigues, A. Kipnis, C. O. Carvalho, M. R. H. Campos, E. C. Monteiro, F. Martins, and T. F. N. Jubé. 2003. Microbiological quality of human milk from a Brazilian milk bank. Rev. Saude Publica 37:775-779.

Trujillo, A. J., M. Capellas, J. Saldo, R. Gervilla, and B. Guamis. 2002. Applications of high hydrostatic pressure on milk and dairy 
products: A review. Innov. Food Sci. Emerg. Technol. 3:295307.

Trujillo, A. J., N. Castro, J. M. Quevedo, A. Argüello, J. Capote, and B. Guamis. 2007. Effect of heat and high-pressure treatments on microbiological quality and immunoglobulin G stability of caprine colostrum. J. Dairy Sci. 90:833-839.

UKAMB. 2009. United Kingdom Association for Milk Banking (UKAMB). http://www.ukamb.org.

Van de Perre, P. 2003. Transfer of antibody via mother's milk. Vaccine 21:3374-3376.

Viazis, S., B. E. Farkas, and J. C. Allen. 2007. Effects of high-pressure processing on immunoglobulin A and lysozyme activity in human milk. J. Hum. Lact. 23:253-261.

Viazis, S., B. E. Farkas, and L. A. Jaykus. 2008. Inactivation of bacterial pathogens in human milk by high-pressure processing. J. Food Prot. 71:109-118.
Walker, W. A. 2002. Development of the intestinal mucosal barrier. J. Pediatr. Gastroenterol. Nutr. 34(Suppl. 1):S33-S39.

Weaver, L. T., H. M. L. Arthur, J. E. G. Bunn, and J. E. Thomas. 1998. Human milk IgA concentrations during the first year of lactation. Arch. Dis. Child. 78:235-239.

WHO. 2003. World Health Organisation. Global Strategy for Infant and Young Child Feeding. Pages 7-8. http://www.who.int/topics/ breastfeeding/en/ Accessed Nov. 21, 2008.

Wight, N. E. 2001. Donor human milk for preterm infants. J. Perinatol. 21:249-254.

Zinkernagel, R. M. 2001. Maternal antibodies, childhood infections, and autoimmune diseases. N. Engl. J. Med. 345:1331-1335. 